PROCEEDINGS OF THE

AMERICAN MATHEMATICAL SOCIETY

Volume 129, Number 10, Pages 3057-3067

S 0002-9939(01)05928-7

Article electronically published on April 16, 2001

\title{
A DIRECT INTEGRAL DECOMPOSITION OF THE WAVELET REPRESENTATION
}

\author{
LEK-HENG LIM, JUDITH A. PACKER, AND KEITH F. TAYLOR
}

(Communicated by David R. Larson)

\begin{abstract}
In this paper we use the concept of wavelet sets, as introduced by $\mathrm{X}$. Dai and D. Larson, to decompose the wavelet representation of the discrete group associated to an arbitrary $n \times n$ integer dilation matrix as a direct integral of irreducible monomial representations. In so doing we generalize a result of F. Martin and A. Valette in which they show that the wavelet representation is weakly equivalent to the regular representation for the Baumslag-Solitar groups.
\end{abstract}

\section{INTRODUCTION}

Several years ago, X. Dai and D. Larson introduced an operator algebraic approach to the study of wavelets, which has proved very useful in the abstract study of both wavelets and frames [6. In particular, their study of local commutant and wavelet sets has provided a great deal of impetus for the research of themselves and others [6, 7, 8].

Also, L. Baggett, both on his own and together with K. Merrill and other collaborators [1, 2] 3], has promoted a representation-theoretic point of view in the study of wavelets and other types of orthonormal bases of $L^{2}\left(\mathbb{R}^{n}\right)$ associated to discrete groups. In [1, he decomposed the Stone-von Neumann representation of the discrete Heisenberg group on $L^{2}(\mathbb{R})$ into a direct integral of representations, and in so doing determined whether or not the translates of Gabor functions parameterized by certain scales spanned a dense subspace of $L^{2}(\mathbb{R})$.

It is our intention in this paper to use the combined methods of Dai and Larson [6] and Baggett [1] to further study the notion of wavelet sets in $\mathbb{R}^{n}$. Recall from [7] that if $A \in M(n, \mathbb{Z}) \cap \mathrm{GL}(n, \mathbb{Q})$ satisfies the condition that all of its eigenvalues have modulus strictly greater than one, it is said to be a dilation matrix. We then can associate to $A$ a unitary dilation operator

$$
D_{A} f(t)=|\operatorname{det} A|^{1 / 2} f(A t),
$$

Received by the editors November 15, 1999 and, in revised form, February 24, 2000.

2000 Mathematics Subject Classification. Primary 65T60, 47N40, 22D20, 22D30; Secondary 22D45, 47L30, 47C05.

Key words and phrases. Wavelet, wavelet set, group representations.

The third author was supported in part by a grant from NSERC Canada.

(C)2001 American Mathematical Society 
and to each $v \in \mathbb{Z}^{n}$ a unitary translation operator

$$
T_{v} f(t)=f(t-v),
$$

defined for every $f \in L^{2}\left(\mathbb{R}^{n}\right)$.

Recall that $\psi \in L^{2}\left(\mathbb{R}^{n}\right)$ is said to be a wavelet for the family of unitary operators $\left\{D_{A}^{m}, T_{v} \mid m \in \mathbb{Z}, v \in \mathbb{Z}^{n}\right\}$ if the family of functions

$$
\left\{D_{A}^{m} T_{v} \psi \mid m \in \mathbb{Z}, v \in \mathbb{Z}^{n}\right\}
$$

forms an orthonormal basis for $L^{2}\left(\mathbb{R}^{n}\right)$. Following Dai and Larson, we define a wavelet set $E$ with respect to the dilation matrix $A$ to be a subset $E \subseteq \mathbb{R}^{n}$ such that the normalization in $L^{2}\left(\mathbb{R}^{n}\right)$ of the characteristic function of $E$, $\mathbf{1}_{E}$, is the Fourier transform of a wavelet with respect to the translation and dilation operators corresponding to $A$. Dai, Larson and Speegle have shown in [7] that fixing an arbitrary $n \times n$ integer dilation matrix $A$, wavelet sets with respect to $A$ always exist, and that we necessarily have $\mu(E)=(2 \pi)^{n}$, for $\mu$ the normalized Lebesgue measure on $\mathbb{R}^{n}$.

Let $\mathcal{G}\left(D_{A}, T_{v}\right)$ denote the group in $\mathcal{U}\left(L^{2}\left(\mathbb{R}^{n}\right)\right)$ generated by the family of operators $\left\{D_{A}, T_{v} \mid v \in \mathbb{Z}^{n}\right\}$. Let $\left\{e_{i} \mid 1 \leq i \leq n\right\}$ denote the standard generators for $\mathbb{Z}^{n}$, so that $\left(e_{i}\right)_{j}=\delta_{i, j}$. Then the following standard commutation relations hold:

$$
T_{e_{i}} D_{A}=D_{A} T_{A e_{i}}=D_{A} T_{\sum_{j=1}^{n} a_{j, i} e_{j}} .
$$

So $\mathcal{G}\left(D_{A}, T_{v}\right)$ can be viewed in various ways. First, it is a subgroup of $\mathcal{U}\left(L^{2}\left(\mathbb{R}^{n}\right)\right)$. Second, it can be viewed as a unitary representation of the discrete group having $n+1$ generators and the following $n+n(n-1) / 2$ relations:

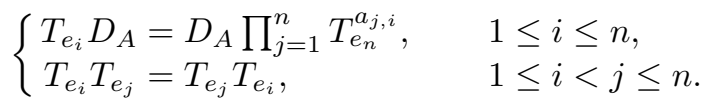

We note that groups with generators satisfying the relations above contain the Baumslag-Solitar groups as a special case; the $C^{*}$-algebras associated to such groups were first observed to be related to the theory of wavelets by B. Brenken in Section 6 of [4]. Finally, letting $\mathbb{Q}_{A}$ denote the subgroup of $\mathbb{Q}^{n}$ defined by

$$
\mathbb{Q}_{A}=\bigcup_{j=0}^{\infty}\left\{A^{-j} v \mid v \in \mathbb{Z}^{n}\right\},
$$

$\mathcal{G}\left(D_{A}, T_{v}\right)$ can be viewed as the image of a representation of the semidirect product of $\mathbb{Q}_{A}$ and $\mathbb{Z}$, which we will write as $\mathbb{Q}_{A} \rtimes_{\vartheta} \mathbb{Z}$. Here for $m \in \mathbb{Z}, \vartheta(m)$ is the automorphism of $\mathbb{Q}_{A}$ defined by

$$
\vartheta(m) \beta=A^{-m} \beta
$$

for $\beta \in \mathbb{Q}_{A}$, and the group operation in the semidirect product is defined by

$$
\left(\beta_{1}, m_{1}\right) \cdot\left(\beta_{2}, m_{2}\right)=\left(\beta_{1}+\vartheta\left(m_{1}\right) \beta_{2}, m_{1}+m_{2}\right)
$$

for $\left(\beta_{i}, m_{i}\right) \in \mathbb{Q}_{A} \rtimes_{\vartheta} \mathbb{Z}$. One easily checks that the correspondence $(\beta, m) \mapsto T_{\beta} D_{A}^{m}$ gives a group isomorphism of $\mathbb{Q}_{A} \rtimes_{\vartheta} \mathbb{Z}$ into $\mathcal{G}\left(D_{A}, T_{v}\right) \subseteq \mathcal{U}\left(L^{2}\left(\mathbb{R}^{n}\right)\right)$. Thus we have a unitary representation of the discrete group $\mathbb{Q}_{A} \rtimes_{\vartheta} \mathbb{Z}$ which we term the wavelet representation of $\mathbb{Q}_{A} \rtimes_{\vartheta} \mathbb{Z}$. We use the following notation for the wavelet representation: for $(\beta, m) \in \mathbb{Q}_{A} \rtimes_{\vartheta} \mathbb{Z}$, let

$$
W(\beta, m)=T_{\beta} D_{A}^{m} .
$$


Then by our remarks above, $W: \mathbb{Q}_{A} \rtimes_{\vartheta} \mathbb{Z} \rightarrow \mathcal{U}\left(L^{2}\left(\mathbb{R}^{n}\right)\right)$ is just the wavelet representation of $\mathbb{Q}_{A} \rtimes_{\vartheta} \mathbb{Z}$.

In this paper, we use the wavelet sets of Dai, Larson and Speegle and modify the techniques of Baggett discussed above to decompose the wavelet representation described above as a direct integral of irreducible representations of $\mathbb{Q}_{A} \rtimes_{\vartheta} \mathbb{Z}$. In so doing we are able to extend and generalize a result of $\mathrm{F}$. Martin and A. Valette [16, in which they showed that certain representations of the Baumslag-Solitar groups, of which the wavelet representation by the univariate wavelet group associated to dilation by 2 is a characteristic example, are weakly equivalent to the regular representations of these groups, hence faithful. Our method will use the existence of the wavelet sets as proved in [7] to decompose the wavelet representation as a direct integral of representations over the wavelet set itself. The main work in the proof then comes as identifying the representations in this direct integral as monomial representations, that is, representations induced from characters on the normal subgroup $\mathbb{Q}_{A}$ of $\mathbb{Q}_{A} \rtimes_{\vartheta} \mathbb{Z}$.

This paper is an offshoot of the first author's M.Sc. thesis done at the National University of Singapore [13]. The third author wishes to thank the Department of Mathematics and the Wavelets Group at the National University of Singapore for their hospitality and support during his visit. Finally, we are grateful to Lawrence Baggett for useful conversations on the proof of Theorem 3.1

\section{USING THE WAVELET SET TO DECOMPOSE THE WAVELET REPRESENTATION}

Let $A$ be a fixed $n \times n$ integer dilation matrix. We first follow the lead of Dai and Larson [6] and study the operators $\left\{D_{A}, T_{v}\right\}$ in the Fourier domain. Let $\mathcal{F}$ denote the Fourier transform on $L^{2}\left(\mathbb{R}^{n}\right)$, i.e.,

$$
\mathcal{F} f(\xi)=(2 \pi)^{-n / 2} \int_{\mathbb{R}^{n}} f(t) e^{-i\langle t, \xi\rangle} d t
$$

for $f \in L^{1} \cap L^{2}\left(\mathbb{R}^{n}\right), t, \xi \in \mathbb{R}^{n}$, and extending to $L^{2}\left(\mathbb{R}^{n}\right)$ (which contains $L^{1} \cap L^{2}\left(\mathbb{R}^{n}\right.$ ) as a dense subset) by the usual limiting process. Hence for $f \in L^{2}\left(\mathbb{R}^{n}\right), \xi \in \mathbb{R}^{n}$, we may define

$$
\widehat{D}_{A} f(\xi)=\mathcal{F} D_{A} \mathcal{F}^{-1} f(\xi)=|\operatorname{det} B|^{-1 / 2} f\left(B^{-1} \xi\right),
$$

where $B=A^{T}$, the matrix transpose of $A$, and

$$
\widehat{T}_{\beta} f(\xi)=\mathcal{F} T_{\beta} \mathcal{F}^{-1} f(\xi)=e^{-i\langle\beta, \xi\rangle} f(\xi),
$$

where $\beta \in \mathbb{Q}_{A}$. As in [6], we calculate the commutant of $\widehat{D}_{A}$ and $\left\{\widehat{T}_{v} \mid v \in \mathbb{Z}^{n}\right\}$ in $\mathcal{B}\left(L^{2}\left(\mathbb{R}^{n}\right)\right)$ :

$$
\left\{\widehat{D}_{A}, \widehat{T}_{v} \mid v \in \mathbb{Z}^{n}\right\}^{\prime}=\left\{M_{g} \mid g \in L^{\infty}\left(\mathbb{R}^{n}\right), g(\xi)=g(B \xi) \text { a.e., } \xi \in \mathbb{R}^{n}\right\},
$$

where $M_{g}$ is the multiplication operator defined by

$$
M_{g} f(\xi)=g(\xi) f(\xi)
$$

for $f \in L^{2}\left(\mathbb{R}^{n}\right), \xi \in \mathbb{R}^{n}$. One obtains (21) as follows: modifying the proof in the one-variable case found in [6], we have

$$
\left\{\widehat{D}_{A}, \widehat{T}_{v} \mid v \in \mathbb{Z}^{n}\right\}^{\prime} \subseteq\left\{\widehat{T}_{\beta} \mid \beta \in \mathbb{Q}_{A}\right\}^{\prime}=\left\{M_{g} \mid g \in L^{\infty}\left(\mathbb{R}^{n}\right)\right\},
$$

and computing $\left\{\widehat{D}_{A}\right\}^{\prime} \cap\left\{M_{g} \mid g \in L^{\infty}\left(\mathbb{R}^{n}\right)\right\}$, one obtains (2). 
Let $E \subseteq \mathbb{R}^{n}$ be a wavelet set for the dilation associated to $A$, so that

$$
\mathcal{F}^{-1}\left(\frac{1}{\sqrt{\mu(E)}} \mathbf{1}_{E}\right)
$$

is a wavelet for $\left\{D_{A}, T_{v}\right\}$, or to use the definition of Dai, Larson and Speegle [7, let $E$ be a transformation wavelet set for the matrix $B=A^{T}$, so that the following conditions are satisfied:

(i) $E$ is a measurable subset of $\mathbb{R}^{n}$, with $B^{j}(E) \cap B^{k}(E)=\emptyset, j \neq k \in \mathbb{Z}$;

(ii) $\mu\left(\mathbb{R}^{n} \backslash \bigcup_{j \in \mathbb{Z}} B^{j}(E)\right)=0$;

(iii) $E$ is translation congruent to the set $[-\pi, \pi)^{n}$ modulo the lattice $\{2 \pi v \mid v \in$ $\left.\mathbb{Z}^{n}\right\}$.

If $E$ satisfies conditions (i) and (ii), we say the $E$ tiles $\mathbb{R}^{n}$ under dilation by $B$ (cf. 8], where such sets are said to be dilation-congruent to $[-\pi, \pi)^{n}$ ). The prototype wavelet set is the subset $E=[-2 \pi,-\pi) \cup[\pi, 2 \pi)$ of $\mathbb{R}$ corresponding to dilation by 2 in $\mathbb{R}$ (see [6]).

Generalizing the earlier results in [6], Dai, Larson and Speegle have shown in [7] 8$]$ that if $A$ is a dilation matrix, then $E$ is a wavelet set for the dilation associated to $A$ if and only if $E$ is a transformation wavelet set for the matrix $B=A^{T}$, and that transformation wavelet sets always exist for an arbitrary $n \times n$ dilation matrices with real-valued entries. We concentrate here on the special case where $A$ has integer entries. It is evident from condition (iii) for transformation matrix sets that any wavelet set $E$ will satisfy $\mu(E)=(2 \pi)^{n}$.

We now are able to state our main theorem:

Theorem 2.1. Let $A$ be an $n \times n$ dilation matrix in $M(n, \mathbb{Z}) \cap \operatorname{GL}(n, \mathbb{Q})$, and let $E \subseteq \mathbb{R}^{n}$ be a transformation wavelet set for the matrix $B=A^{T}$. Then the wavelet representation $W$ of $\mathbb{Q}_{A} \rtimes_{\vartheta} \mathbb{Z}$ defined in (1D) is unitarily equivalent to a direct integral of representations $\left\{\widetilde{W}_{x} \mid x \in E\right\}$, where each $\widetilde{W}_{x}$ is an irreducible monomial representation induced from a character on the normal abelian subgroup $\mathbb{Q}_{A} \rtimes_{\vartheta}\{0\}$ of $\mathbb{Q}_{A} \rtimes_{\vartheta} \mathbb{Z}$. Indeed, for each $x \in E$, the representation $\widetilde{W}_{x}: \mathbb{Q}_{A} \rtimes_{\vartheta} \mathbb{Z} \rightarrow \mathcal{U}\left(l^{2}(\mathbb{Z})\right)$ is defined by

$$
\left[\widetilde{W}_{x}(\beta, m) g\right](k)=e^{-i\left\langle x, A^{k} \beta\right\rangle} g(k-m)
$$

for $x \in E, k \in \mathbb{Z}$ and $g \in l^{2}(\mathbb{Z})$.

We will prove Theorem 2.1 in several stages. We first construct another Hilbert space isomorphism, which will make the situation more transparent. Since the sets $B^{k}(E), k \in \mathbb{Z}$, tile $\mathbb{R}^{n}$ a.e., we can define measurable maps

$$
\pi: \mathbb{R}^{n} \backslash\{0\} \rightarrow E \quad \text { and } \quad p: \mathbb{R}^{n} \backslash\{0\} \rightarrow \mathbb{Z}
$$

almost everywhere by the formula

$$
B^{-p(\xi)} \pi(\xi)=\xi
$$

i.e., $\pi(\xi)$ is the unique element of $E$ which is $B$-dilation congruent to $\xi$, and $p(\xi)$ is the unique element of $\mathbb{Z}$ such that $B^{p(\xi)} \xi \in E$. For example, if $\xi \in E, \pi(\xi)=\xi$ and $p(\xi)=0$, if $\xi \in B^{-1}(E), \pi(\xi)=B \xi$ and $p(\xi)=1$, etc.

Having thus defined the maps $\pi$ and $p$ a.e. on $\mathbb{R}^{n}$, we now define a measurable transformation $\varphi: \mathbb{R}^{n} \rightarrow E \times \mathbb{Z}$ a.e. on $\mathbb{R}^{n}$ by

$$
\varphi(\xi)=(\pi(\xi), p(\xi)) .
$$


Finally, we let $\Phi: L^{2}\left(\mathbb{R}^{n}\right) \rightarrow L^{2}(E \times \mathbb{Z})$ be the Hilbert space isomorphism defined by

$$
\Phi f(x, k)=|\operatorname{det} B|^{k / 2} f\left(B^{k} x\right)
$$

for $f \in L^{2}(E \times \mathbb{Z})$. One readily checks that $\Phi$ preserves norms and its inverse $\Phi^{-1}: L^{2}(E \times \mathbb{Z}) \rightarrow L^{2}\left(\mathbb{R}^{n}\right)$ is defined by

$$
\Phi^{-1} f(\xi)=|\operatorname{det} B|^{p(\xi) / 2} f(\pi(\xi),-p(\xi))
$$

for $f \in L^{2}\left(\mathbb{R}^{n}\right)$ and $\xi \in \mathbb{R}^{n}$, so that $\Phi$ and $\Phi^{-1}$ are Hilbert space isomorphisms.

We now define maps $\widetilde{D}_{A}=\Phi \widehat{D}_{A} \Phi^{-1}$, and $\widetilde{T}_{\beta}=\Phi \widehat{T}_{\beta} \Phi^{-1}, \beta \in \mathbb{Q}_{A}$, mapping the Hilbert space $L^{2}(E \times \mathbb{Z})$ to itself, and calculate

$$
\begin{aligned}
\widetilde{D}_{A} f(x, k) & =\Phi\left(\widehat{D}_{A}\left(\Phi^{-1} f\right)\right)(x, k) \\
& =|\operatorname{det} B|^{k / 2} \widehat{D}_{A}\left(\Phi^{-1} f\right)\left(B^{k} x\right) \\
& =|\operatorname{det} B|^{k / 2}|\operatorname{det} B|^{-k / 2} f(x, k-1) \\
& =f(x, k-1)
\end{aligned}
$$

and

$$
\begin{aligned}
\widetilde{T}_{\beta} f(x, k) & =\Phi\left(\widehat{T}_{\beta}\left(\Phi^{-1} f\right)\right)(x, k) \\
& =|\operatorname{det} B|^{k / 2} \widehat{T}_{\beta}\left(\Phi^{-1} f\right)\left(B^{k} x\right) \\
& =|\operatorname{det} B|^{k / 2} e^{-i\left\langle\beta, B^{k} x\right\rangle}|\operatorname{det} B|^{-k / 2} f(x, k) \\
& =e^{-i\left\langle\beta, B^{k} x\right\rangle} f(x, k) \\
& =e^{-i\left\langle x, A^{k} \beta\right\rangle} f(x, k),
\end{aligned}
$$

where $f \in L^{2}(E \times \mathbb{Z})$.

We have thus constructed a new unitary representation of the wavelet group $\mathbb{Q}_{A} \rtimes_{\vartheta} \mathbb{Z}$ on the Hilbert space $L^{2}(E \times \mathbb{Z})$ which is unitarily equivalent to the representation $W$ defined in (11). We denote this representation as follows:

$$
\widetilde{W}(\beta, m)=\widetilde{T}_{\beta} \widetilde{D}_{A}^{m}
$$

for $(\beta, m) \in \mathbb{Q}_{A} \rtimes_{\vartheta} \mathbb{Z}$. One computes that

$$
[\widetilde{W}(\beta, m) f](x, k)=e^{-i\left\langle x, A^{k} \beta\right\rangle} f(x, k-m)
$$

for $f \in L^{2}(E \times \mathbb{Z})$ and $(\beta, m) \in \mathbb{Q}_{A} \rtimes_{\vartheta} \mathbb{Z}$. For fixed $x \in E$, the formula in (5) is given exactly by the formula for the representation $\widetilde{W}_{x}$ given in the statement of Theorem 2.1. We now prove that the representations $\widetilde{W}_{x}$ are monomial.

Proposition 2.2. Let $E$ be as in the statement of Theorem 2.1, and for each $x \in E$ let $\widetilde{W}_{x}$ be the representation of $\mathbb{Q}_{A} \rtimes_{\vartheta} \mathbb{Z}$ on the Hilbert space $l^{2}(\mathbb{Z})$ defined by

$$
\left[\widetilde{W}_{x}(\beta, m) g\right](k)=e^{-i\left\langle x, A^{k} \beta\right\rangle} g(k-m) .
$$

Then for every $x \in E, \widetilde{W}_{x}$ is an irreducible monomial representation of $\mathbb{Q}_{A} \rtimes_{\vartheta} \mathbb{Z}$, that is, each $\widetilde{W}_{x}$ is an irreducible representation which is induced from the onedimensional representation $\chi_{x}$ on the normal abelian subgroup $\mathbb{Q}_{A} \rtimes_{\vartheta}\{0\} \cong \mathbb{Q}_{A}$ defined by

$$
\chi_{x}(\beta)=e^{-i\langle x, \beta\rangle}
$$

for $\beta \in \mathbb{Q}_{A}$. 
Proof. We first note that we have inclusions $\mathbb{Q}_{A} \subseteq \mathbb{Q}^{n} \subseteq \mathbb{R}^{n}$ which give us an inclusion monomorphism $\iota: \mathbb{Q}_{A} \rightarrow \mathbb{R}^{n}$. Hence by Pontryagin duality we have a dual homomorphism $\widehat{\iota}: \widehat{\mathbb{R}}^{n} \cong \mathbb{R}^{n} \rightarrow \widehat{\mathbb{Q}}_{A}$, where the identification between $\mathbb{R}^{n}$ and $\widehat{\mathbb{R}}^{n}$ is given by $\xi \mapsto \chi_{\xi}(\cdot)=e^{-i\langle\xi, \cdot\rangle}$, and we easily check that for $x \in \mathbb{R}^{n}$, we have $\widehat{\iota}(x)=\chi_{x}$ as defined in the statement of the proposition. Now the action $\vartheta$ of $\mathbb{Z}$ on $\mathbb{Q}_{A}$ via automorphisms again via duality gives us a dual action $\widehat{\vartheta}$ of $\mathbb{Z}$ on $\widehat{\mathbb{Q}}_{A}$, defined by $\widehat{\vartheta}(\chi)=\chi \circ \vartheta, \chi \in \widehat{\mathbb{Q}}_{A}$. By the theory of group representations for semi-direct product groups (for a reference, see [15]), it is known that for every $\chi \in \widehat{\mathbb{Q}}_{A}$, and for every $m \in \mathbb{Z}$, the induced representations $\operatorname{Ind}_{\mathbb{Q}_{A} \rtimes_{\vartheta} \mathbb{Z}}(\chi)$ and $\left.\operatorname{Ind}_{\mathbb{Q}_{A} \rtimes_{\vartheta} \mathbb{Z}}^{\mathbb{Q}_{\vartheta^{m}}}(\chi)\right)$ are equivalent, and that for fixed $\chi \in \widehat{\mathbb{Q}}_{A}, \operatorname{Ind}_{\mathbb{Q}_{A} \rtimes_{\vartheta} \mathbb{Z}}^{\mathbb{Q}_{A}}(\chi)$ is irreducible if and only if $\widehat{\vartheta}^{m}(\chi) \neq \chi$ for every $m \in \mathbb{Z}$. Now in our case, for $x \in E$ and $m \in \mathbb{Z}$, we have

$$
\left[\widehat{\vartheta}^{m}\left(\chi_{x}\right)\right](\beta)=\chi_{x}\left(\vartheta^{m}(\beta)\right)=e^{-i\left\langle x, A^{-m} \beta\right\rangle}=e^{-i\left\langle B^{-m} x, \beta\right\rangle}=\chi_{B^{-m} x}(\beta) .
$$

Since $x \in E$, which is a transformation wavelet set for $B$, we know that the sets $\left\{B^{m}(E) \mid m \in \mathbb{Z}\right\}$ are pairwise disjoint. Hence $B^{-m} x \neq x$ for every $m \in \mathbb{Z}$. It follows that for every $x \in E$, the representation $\operatorname{Ind}_{\mathbb{Q}_{A} \rtimes_{\vartheta} \mathbb{Z}}^{\mathbb{Q}_{A}}\left(\chi_{x}\right)$ is irreducible.

We now show that $\operatorname{Ind}_{\mathbb{Q}_{A} \rtimes_{\vartheta} \mathbb{Z}}^{\mathbb{Q}_{A}}\left(\chi_{x}\right)$ is equivalent to the representation $\widetilde{W}_{x}$ given in the statement of Theorem 2.1 Here we use the construction of induced representations given in Chapter $\mathrm{X}$ of [12]. In this construction, for a locally compact group $G$ with closed subgroup $H$, one first needs a Borel cross section $c: H \backslash G \rightarrow G$. Here, $G=\mathbb{Q}_{A} \rtimes_{\vartheta} \mathbb{Z}, H=\mathbb{Q}_{A} \rtimes_{\vartheta}\{0\} \cong \mathbb{Q}_{A}$, and the (right) coset space $H \backslash G \cong \mathbb{Z}$. Furthermore the groups are discrete, and our cross section $c$ is actually a group isomorphism defined by

$$
c(k)=(0, k) \in \mathbb{Q}_{A} \rtimes_{\vartheta} \mathbb{Z}
$$

for $k \in \mathbb{Z}$. We now use $c$ to define a one-cocycle $\omega$ for the right action of $G$ on the coset space $H \backslash G$ in the standard fashion: $\omega: H \backslash G \times G \rightarrow H \cong \mathbb{Q}_{A}$ is defined by

$$
\begin{aligned}
\omega(k,(\beta, m)) & =c(k)(\beta, m)[c(k \cdot(\beta, m))]^{-1} \\
& =(0, k)(\beta, m)(0,-k-m) \\
& =\left(A^{-k} \beta, k+m\right)(0,-k-m) \\
& =\left(A^{-k} \beta, 0\right)
\end{aligned}
$$

for $k \in H \backslash G \cong \mathbb{Z}$ and $(\beta, m) \in \mathbb{Q}_{A} \rtimes_{\vartheta} \mathbb{Z}$. Then we recall from Chapter $\mathrm{X}$ of [12] that given a unitary representation $\Lambda$ of the group $H$ on the Hilbert space $\mathcal{H}$, the induced representation $\operatorname{Ind}_{H}^{G}(\Lambda)$ has as its representation space $L^{2}(H \backslash G) \otimes \mathcal{H} \cong$ $L^{2}(H \backslash G, \mathcal{H})$ where the measure on $H \backslash G$ is quasi-invariant under translation by $G$. If this measure is invariant, then the formula for $\operatorname{Ind}_{H}^{G}(\Lambda)$ is given by

$$
\left[\operatorname{Ind}_{H}^{G}(\Lambda)(x) f\right](\bar{y})=[\Lambda(\omega(\bar{y}, x)) f](\bar{y} x)
$$

for $x \in G, \bar{y} \in H \backslash G, f \in L^{2}(H \backslash G, \mathcal{H})$. Here all our groups are discrete, and the translation-invariant measure on the coset space $H \backslash G \cong \mathbb{Z}$ is just the counting measure. Hence for $x \in E$ and $\chi_{x}$ defined as in the statement of Proposition 2.2 we have $\mathcal{H}=\mathbb{C}$ and $L^{2}(H \backslash G, \mathcal{H}) \cong l^{2}(\mathbb{Z})$, so that with respect to these identifications,

$$
\begin{aligned}
\left.\operatorname{Ind}_{\mathbb{Q}_{A}}^{\mathbb{Q}_{A} \rtimes \mathbb{Z}}\left(\chi_{x}\right)(\beta, m) f\right](k) & =\chi_{x}(\omega(k,(\beta, m))) f(k \cdot(\beta, m)) \\
& =e^{-i\left\langle x, A^{-k} \beta\right\rangle} f(k+m)
\end{aligned}
$$


for $k \in \mathbb{Z},(\beta, m) \in \mathbb{Q}_{A} \rtimes_{\vartheta} \mathbb{Z}$ and $f \in l^{2}(\mathbb{Z})$. Now this is not exactly the same as our formula for $\widetilde{W}_{x}$, but defining the unitary involution $V: l^{2}(\mathbb{Z}) \rightarrow l^{2}(\mathbb{Z})$ by the formula $V f(k)=f(-k)$, one easily checks that

$$
V^{-1}\left[\widetilde{W}_{x}(\beta, m)\right] V=\operatorname{Ind}_{\mathbb{Q}_{A} \rtimes_{\vartheta} \mathbb{Z}}^{\mathbb{Q}_{A}}\left(\chi_{x}\right)(\beta, m)
$$

for $(\beta, m) \in \mathbb{Q}_{A} \rtimes_{\vartheta} \mathbb{Z}$ and $x \in E$. This completes the proof of the proposition.

We are now ready to finish the proof of Theorem 2.1.

Proof of Theorem [2.1. We have constructed an equivalence between the wavelet representation $W$ of (11) and the representation $\widetilde{W}$ of (4), so it only remains to show that $\widetilde{W}$ is a direct integral of irreducible monomial representations. Now in general the theory of direct integrals of measurable fields of Hilbert spaces and direct integrals of unitary representations is very technical (see, for example, [15] for a reference). But in our situation, the representation space for the representation $\widetilde{W}$ is $L^{2}(E \times \mathbb{Z})$ which can also be viewed as $L^{2}(E) \otimes l^{2}(\mathbb{Z})$, and there are no technicalities involved in showing that this last Hilbert space is exactly the direct integral $\int_{E}^{\oplus}\left(l^{2}(\mathbb{Z})\right)_{x} d x$. With respect to this decomposition of $L^{2}(E \times \mathbb{Z})$ it is clear that the decomposition of $\widetilde{W}$ over the measurable subset $E$ is given exactly by the representations $\left\{\widetilde{W}_{x} \mid x \in E\right\}$ defined in (31). Finally, Proposition [2.2] has shown that for each $x \in E$ the representation $\widetilde{W}_{x}$ is an irreducible monomial representation of $\mathbb{Q}_{A} \rtimes_{\vartheta} \mathbb{Z}$, and the proof of Theorem 2.1 is complete.

Remark 2.3. We note here that to prove Theorem 2.1 we did not need to use all of the properties (i)-(iii) of transformation wavelet sets. Indeed, all we really used were properties (i) and (ii): we did not need that $E$ be $2 \pi$-translation congruent to $[-\pi, \pi)^{n}$. The pairwise disjointness of the sets $\left\{B^{j}(E) \mid j \in \mathbb{Z}\right\}$ and the fact that $\mu\left(\mathbb{R}^{n} \backslash \bigcup_{j \in \mathbb{Z}} B^{j}(E)\right)=0$ allowed us to construct the isomorphism of measure spaces $\varphi: \mathbb{R}^{n} \rightarrow E \times \mathbb{Z}$ which was crucial in the direct integral procedure. Property (ii) ensures that the representations $\widetilde{W}_{x}$ are irreducible. It is only when one wants to show that $\mathcal{F}^{-1}\left(\mathbf{1}_{E} / \sqrt{\mu(E)}\right)$ is a wavelet for the dilation system $\left\{D_{A}, T_{v} \mid v \in \mathbb{Z}^{n}\right\}$ that property (iii) becomes essential. The next corollary gives a proof of this result, originally due to Dai and Larson ([6], cf. Lemmas 2.2 and 2.3) which uses the framework that we have set up. The main difference between our method and that of Dai and Larson is the slightly more transparent presentation given to the operators $\left\{\widetilde{D}_{A}^{m} \mid m \in \mathbb{Z}\right\}$ as operators which are just translations in the second variable on our realization of the Hilbert space as $L^{2}(E \times \mathbb{Z})$.

Corollary 2.4. Let $A$ be a dilation matrix in $M(n, \mathbb{Z}) \cap \mathrm{GL}(n, \mathbb{Q})$ with $A^{T}=B$, and suppose that $E$ is a subset of $\mathbb{R}^{n}$ with finite measure such that the sets $\left\{B^{j}(E) \mid j \in\right.$ $\mathbb{Z}\}$ are pairwise disjoint and $\mu\left(\mathbb{R}^{n} \backslash \bigcup_{j \in \mathbb{Z}} B^{j}(E)\right)=0$. Then $\psi=\mathcal{F}^{-1}\left(\mathbf{1}_{E} / \sqrt{\mu(E)}\right)$ is a wavelet for the system $\left\{D_{A}, T_{v} \mid v \in \mathbb{Z}^{n}\right\}$ if and only if the sets $\{E+2 \pi v \mid v \in$ $\left.\mathbb{Z}^{n}\right\}$ are pairwise disjoint up to sets of measure zero and $\mu\left(\mathbb{R}^{n} \backslash \bigcup_{v \in \mathbb{Z}^{n}}(E+2 \pi v)\right)=$ 0 .

Proof. The content of Remark 2.3 shows that under the hypotheses of the Corollary, the proof of Theorem 2.1 still works. Keeping the notation of Theorem 2.1 and Proposition 2.2, we see that the set $\left\{D_{A}^{m} T_{v} \psi \mid m \in \mathbb{Z}, v \in \mathbb{Z}^{n}\right\}$ will be an orthonormal basis for $L^{2}\left(\mathbb{R}^{n}\right)$ if and only if the set $\left\{\widetilde{D}_{A}^{m} \widetilde{T}_{v} \Phi^{-1}\left(\mathbf{1}_{E} / \sqrt{\mu(E)}\right) \mid m \in \mathbb{Z}, v \in \mathbb{Z}^{n}\right\}$ 
is an orthonormal basis for $L^{2}(E \times \mathbb{Z})$. Now

$$
\Phi^{-1}\left(\frac{1}{\sqrt{\mu(E)}} \mathbf{1}_{E}\right)=\frac{1}{\sqrt{\mu(E)}} \mathbf{1}_{E \times\{0\}},
$$

where $E \times\{0\}$ is viewed as a subset of $E \times \mathbb{Z}$, and since $\widetilde{D}_{A}^{m} f(x, k)=f(x, k-m)$ it is clear that for any $f \in L^{2}(E \times \mathbb{Z})$ which is supported on $E \times\{0\}$, the function $\widetilde{D}_{A}^{m} f$ will be supported on $E \times\{m\}, m \in \mathbb{Z}$. It follows from this and an inspection of the formula for $\widetilde{D}_{A}$ that if $\left\{f_{i} \mid i \in\right.$ index set $\left.I\right\}$ is a set of orthonormal functions whose support lies in $E \times\{0\}$ which form a basis for $L^{2}(E \times\{0\})$, then the set $\left\{\widetilde{D}_{A}^{m} f_{i} \mid m \in \mathbb{Z}, i \in I\right\}$ will form an orthonormal basis for $L^{2}(E \times \mathbb{Z})$. Thus in order to obtain the results of the corollary it is enough to show that the stated conditions on $E$ are necessary and sufficient to ensure that the set $\left\{\widetilde{T}_{v}\left(\mathbf{1}_{E \times\{0\}} / \sqrt{\mu(E)}\right) \mid v \in\right.$ $\left.\mathbb{Z}^{n}\right\}$ is an orthonormal basis for $L^{2}(E \times\{0\})$. Recall that for $v \in \mathbb{Z}^{n}$ we have

$$
\widetilde{T}_{v}\left(\frac{1}{\sqrt{\mu(E)}} \mathbf{1}_{E \times\{0\}}\right)(x, 0)=\frac{1}{\sqrt{\mu(E)}} e^{-i\langle v, x\rangle}
$$

for $x \in E$ and it is well known (cf. [6] Lemma 4.2, Lemma 4.3, [8]) that as $v$ varies over all of $\mathbb{Z}^{n}$, the functions above will give an orthonormal basis for $L^{2}(E)$ if and only if $E$ is translation congruent to $[-\pi, \pi)^{n}$, which is equivalent to the conditions on $E$ given in the statement of the corollary.

\section{WEAK EQUIVALENCE OF THE WAVELET REPRESENTATION AND THE REGULAR REPRESENTATION}

Let $d \in \mathbb{N}$, and for $d \geq 2$ let $B S_{d}$ denote the Baumslag-Solitar groups considered by F. Martin and A. Valette [16]; we recall that $B S_{d}$ has two generators $a$ and $b$ satisfying the relation $a b a^{-1}=b^{d}$. It is clear that with respect to our notation, $B S_{d}$ is the wavelet group corresponding to the dilation $A=(d)$ on $\mathbb{R}^{1}$. In Theorem 8 of [16], it is shown that the regular representation of $B S_{d}$ on $l^{2}\left(B S_{d}\right)$ and the wavelet representation of $B S_{d}$ on $L^{2}(\mathbb{R})$ are weakly equivalent. The proof of our Theorem 2.1 allows us to extend this result to our wider class of semidirect product wavelet groups $\mathbb{Q}_{A} \rtimes_{\vartheta} \mathbb{Z}$, where as usual for $n \geq 1, A$ is a dilation matrix in $M(n, \mathbb{Z}) \cap$ $\operatorname{GL}(n, \mathbb{Q}), \mathbb{Q}_{A}$ is the subgroup $\left\{A^{m} v \mid m \in \mathbb{Z}, v \in \mathbb{Z}^{n}\right\}$ of $\mathbb{Q}^{n}$ determined by $A$, and $\vartheta$ is the automorphism of $\mathbb{Q}_{A}$ associated to $A$. We thank Lawrence Baggett for suggesting that we use the reference [10, which allowed us to considerably simplify our original proof.

Theorem 3.1. Let $n \in \mathbb{N}$ and let $A$ be a dilation matrix in $M(n, \mathbb{Z}) \cap \operatorname{GL}(n, \mathbb{Q})$. Let $\mathbb{Q}_{A} \rtimes_{\vartheta} \mathbb{Z}$ be the wavelet group associated to these parameters described in Section 2 . Then the regular representation and the wavelet representation of $\mathbb{Q}_{A} \rtimes_{\vartheta} \mathbb{Z}$ are weakly equivalent. Hence the wavelet representation of $\mathbb{Q}_{A} \rtimes_{\vartheta} \mathbb{Z}$ is faithful.

Proof. Let $E$ be a transformation wavelet set for $B=A^{T}$. The proof of Theorem 2.1 shows that the wavelet representation $W$ is unitarily equivalent to a representation $\widetilde{W}$ which can be expressed as a direct integral

$$
\int_{E}^{\oplus} \widetilde{W_{x}} d x
$$

where for each $x \in E, \widetilde{W}_{x}$ is an irreducible representation which is unitarily equivalent to $\operatorname{Ind}_{\mathbb{Q}_{A}}^{\mathbb{Q}_{A} \rtimes_{\vartheta} \mathbb{Z}}\left(\chi_{x}\right)$ defined on the Hilbert space $l^{2}(\mathbb{Z})$. We also noted in the proof 
of Proposition 2.2 that for $x \in E$ and $m \in \mathbb{Z}$ the representations $\operatorname{Ind}_{\mathbb{Q}_{A}{ }^{\mathbb{Q}_{\vartheta}} \mathbb{Z}}\left(\chi_{x}\right)$ and

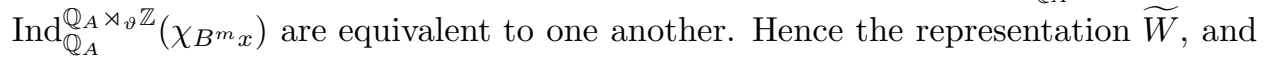
consequently the wavelet representation $W$, is weakly contained in the set of all of the monomial representations

$$
\left\{\operatorname{Ind}_{\mathbb{Q}_{A}}^{\mathbb{Q}_{A} \rtimes_{\vartheta} \mathbb{Z}}\left(\chi_{y}\right) \mid y \in \bigcup_{j \in \mathbb{Z}} B^{j}(E)\right\} .
$$

Let us now consider the regular representation $R$ of $\mathbb{Q}_{A} \rtimes_{\vartheta} \mathbb{Z}$. Recall that $R$ itself is an induced representation,

$$
R=\operatorname{Ind}_{\{1\}}^{\mathbb{Q}_{A} \rtimes_{\vartheta} \mathbb{Z}}(1),
$$

where here $\{1\}$ represents the trivial subgroup of $\mathbb{Q}_{A} \rtimes_{\vartheta} \mathbb{Z}$, and the representation of $\{1\}$ we are inducing is the trivial one-dimensional representation on $\mathbb{C}$. By the theory of induction in stages [15], we can write

$$
R \cong \operatorname{Ind}_{\mathbb{Q}_{A} \mathbb{Q}_{A} \rtimes \mathbb{Z}}\left[\operatorname{Ind}_{\{1\}}^{\mathbb{Q}_{A}}(1)\right] .
$$

Now let $R_{1}$ denote the regular representation of the discrete group $\mathbb{Q}_{A}$, that is,

$$
R_{1}=\operatorname{Ind}_{\{1\}}^{\mathbb{Q}_{A}}(1)
$$

By the Fourier theory connecting compact and discrete abelian groups, $R_{1}$ is equivalent to a representation on the space $L^{2}\left(\widehat{\mathbb{Q}}_{A}\right)$ and in fact can be represented as a direct integral of characters as follows. Setting $\widehat{\mathbb{Q}}_{A}=\Sigma_{A}$, we have

$$
R_{1} \cong \int_{\Sigma_{A}}^{\oplus} \gamma d \gamma
$$

where each $\gamma \in \Sigma_{A}$, being a character of $\mathbb{Q}_{A}$, is exactly a one-dimensional representation on the space $\mathbb{C}$. We thus obtain that $R$ is equivalent to

$$
\operatorname{Ind}_{\mathbb{Q}_{A} \mathbb{Q} \vartheta_{\vartheta} \mathbb{Z}}\left[\int_{\Sigma_{A}}^{\oplus} \gamma d \gamma\right]
$$

and since the processes of taking direct integrals of representations and inducing from a subgroup commute, we have that

$$
R \cong \int_{\Sigma_{A}}^{\oplus}\left[\operatorname{Ind}_{\mathbb{Q}_{A} \rtimes_{\vartheta} \mathbb{Z}}^{\mathbb{Q}_{A}}(\gamma)\right] d \gamma
$$

the representation on the right-hand side being defined on the Hilbert space

$$
\int_{\Sigma_{A}}^{\oplus}\left[l^{2}(\mathbb{Z})\right]_{\gamma} d \gamma
$$

Since we have already shown in the proof of Proposition 2.2 that

$$
\left\{\chi_{y} \mid y \in \bigcup_{j \in \mathbb{Z}} B^{j}(E)\right\} \subseteq\left\{\chi_{t} \mid t \in \mathbb{R}^{n}\right\} \subseteq \Sigma_{A}=\widehat{\mathbb{Q}}_{A},
$$

we have shown directly that the representation $\widetilde{W}$ and hence the wavelet representation $W$ is weakly contained in $R$. Of course, as with the Baumslag-Solitar groups studied in [16], this also follows immediately from the fact that our group $\mathbb{Q}_{A} \rtimes_{\vartheta} \mathbb{Z}$, being the semi-direct product of two abelian groups, is amenable.

To show that $R$ is weakly contained in $W$, we first note that our hypotheses on $B$ together with Proposition 2.4 of 4 ] show that if we let $\widehat{\iota}: \mathbb{R}^{n} \rightarrow \Sigma_{A}$ be the monomorphism constructed in the proof of Proposition 2.2. then the range of $\widehat{\iota}$ is dense in $\Sigma_{A}$. Since $\mu\left(\mathbb{R}^{n} \backslash \bigcup_{j \in \mathbb{Z}} B^{j}(E)\right)=0, \bigcup_{j \in \mathbb{Z}} B^{j}(E)$ is dense in $\mathbb{R}^{n}$ in the 
usual topology, so that $\widehat{\iota}\left(\bigcup_{j \in \mathbb{Z}} B^{j}(E)\right)$ is dense in $\widehat{\iota}\left(\mathbb{R}^{n}\right)$ in the relative topology. It thus follows that $\widehat{\imath}\left(\bigcup_{j \in \mathbb{Z}} B^{j}(E)\right)$ is dense in $\Sigma_{A}$. Hence

$$
\overline{\widehat{\imath}\left(\bigcup_{j \in \mathbb{Z}} B^{j}(E)\right)}=\overline{\left\{\chi_{y} \mid y \in \bigcup_{j \in \mathbb{Z}} B^{j}(E)\right\}}=\Sigma_{A} .
$$

The argument that now follows is similar to the proof of Lemma 4 in [16, which was not used in the proof of Theorem 8 of [16]. We now use the continuity of the induction process (cf. [10]) to deduce that

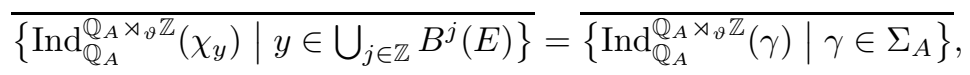

where the closure is taken in the hull-kernel topology. We have already shown that $W$ is unitarily equivalent to the direct integral of the representations

$$
\left\{\operatorname{Ind}_{\mathbb{Q}_{A}}^{\mathbb{Q}_{A} \rtimes_{\vartheta} \mathbb{Z}}\left(\chi_{y}\right) \mid y \in E\right\},
$$

and that for fixed $y \in E$ and $m \in \mathbb{Z}, \operatorname{Ind}_{\mathbb{Q}_{A} \rtimes_{\vartheta} \mathbb{Z}}^{\mathbb{Q}_{1}}\left(\chi_{B^{m} y}\right)$ is unitarily equivalent to the representation $\operatorname{Ind}_{\mathbb{Q}_{A} \rtimes_{\vartheta} \mathbb{Z}}^{\mathbb{Q}_{A}}\left(\chi_{y}\right)$. Since $R$ is the direct integral of the representations $\operatorname{Ind}_{\mathbb{Q}_{A} \rtimes_{\vartheta} \mathbb{Z}}^{\mathbb{Q}_{(}}(\gamma)$, it follows from (6) that $R$ is weakly contained in $W$, so that $W$ and $R$ are weakly equivalent. Finally, as noted in [16], $R$ is faithful since $\mathbb{Q}_{A} \rtimes_{\vartheta} \mathbb{Z}$ is amenable, and it follows from the definition of weak equivalence that $W$ is faithful, thus generalizing Theorem 8 proved in [16] to the wider class of groups $\mathbb{Q}_{A} \rtimes_{\vartheta} \mathbb{Z}$.

\section{REFERENCES}

[1] L. Baggett, "Processing a radar signal and representations of the discrete Heisenberg group," Colloq. Math., LX/LXI (1), 1990, pp. 195-203. MR 92h:22014

[2] L. Baggett, A. Carey, W. Moran and P. Ohring, "General existence theorems for orthonormal wavelets, an abstract approach," Publ. Res. Inst. Math. Sci., 31 (1), 1995, pp. 95-111. MR 96c: 42060

[3] L. Baggett, H. Medina and K. Merrill, "Generalized multiresolution analyses, and a construction procedure for all wavelet sets in $\mathbb{R}^{n}, "$ J. Fourier Anal. Appl. 5, 1999, pp. 563-573. CMP 2000:11

[4] B. Brenken, "The local product structure of expansive automorphisms of solenoids and their associated $C^{*}$-algebras," Canad. J. Math., 48 (4), 1996, pp. 692-709. MR 98c:22003

[5] B. Brenken and P. Jorgensen, "A family of dilation crossed product algebras," J. Operator Theory, 25, 1991, pp. 299-308. MR 94m:46103

[6] X. Dai and D.R. Larson, "Wandering vectors for unitary systems and orthogonal wavelets," Mem. Amer. Math. Soc., 134 (640), July 1998. MR 98m:47067

[7] X. Dai, D.R. Larson and D. Speegle, "Wavelet sets in $\mathbb{R}^{n}$," J. Fourier Anal. Appl., 3 (4), 1997, pp. 451-456. MR 98m:42048

[8] X. Dai, D.R. Larson and D. Speegle, "Wavelet sets in $\mathbb{R}^{n}$ II," pp. 15-40, Wavelets, Multiwavelets, and their Applications, Contemp. Math., 216, American Mathematical Society, Providence, RI, 1998. MR 99d:42054

[9] I. Daubechies, Ten Lectures on Wavelets, CBMS-NSF Regional Conference Series in Applied Mathematics, 61, SIAM, Philadelphia, PA, 1992. MR 93e:42045

[10] J.M.G. Fell, "Weak containment and induced representations of groups," Canadian J. Math., 14, 1962, pp. 237-268. MR 27:242

[11] D. Han and D.R. Larson, "Frames, bases and group representations," Mem. Amer. Math. Soc., 147, 2000. MR 2001a:47013

[12] A.A. Kirillov, Elements of the Theory of Representations, Grundlehren der mathematischen Wissenschaften, 220, Springer-Verlag, Berlin Heidelberg, 1976. MR 54:447

[13] L.-H. Lim, "Group Representations, Operator Algebras and Wavelets," M.Sc. thesis, National University of Singapore, 1998.

[14] G.W. Mackey, "Induced representations of locally compact groups I," Ann. of Math., 55 (2), 1952, pp. 101-139. MR 13:434a 
[15] G.W. Mackey, The Theory of Unitary Group Representations, Chicago Lectures in Mathematics, University of Chicago Press, Chicago, IL, 1976. MR 53:686

[16] F. Martin and A. Valette, "Markov operators on the solvable Baumslag-Solitar groups," Experiment. Math., 9, 2000, pp. 291-300. CMP 2000:17

Department of Mathematics, Malott Hall, Cornell University, Ithaca, New York 14853-4201

E-mail address: lekheng@math.cornell.edu

Current address: Department of Pure Mathematics and Mathematical Statistics, University of Cambridge, Wilberforce Road, Cambridge CB3 0WA, United Kingdom

Department of Mathematics, National University of Singapore, 10 Kent Ridge CresCEnT, Singapore 119260

E-mail address: matjpj@leonis.nus.edu.sg

Department of Mathematics and Statistics, University of Saskatchewan, 106 Wiggins Road, Saskatoon, Saskatchewan, Canada S7N 5E6

E-mail address: taylor@math.usask.ca 